\title{
Up to Date Management of DCIS and Future Directions
}

\author{
Rodrigo Arrangoiz , Fernando Cordera, Eduardo Moreno, Enrique Luque-de-Leon, Manuel Muñoz \\ Sociedad Quirúrgica S.C. at the American British Cowdray Medical Center, Colonia Tlaxala, Delegación Cuajimalpa, Mexico City, \\ Mexico \\ Email: ^rodrigo.arrangoiz@gmail.com, fernandocordera@gmail.com, morenomd7@icloud.com, enrique.luque.dl@gmail.com, \\ munozmd1@gmail.com
}

How to cite this paper: Arrangoiz, R., Cordera, F., Moreno, E., Luque-de-Leon, E. and Muñoz, M. (2020) Up to Date Management of DCIS and Future Directions. Advances in Breast Cancer Research, 9, 78-100.

https://doi.org/10.4236/abcr.2020.93007

Received: May 18, 2020

Accepted: June 15, 2020

Published: June 18, 2020

Copyright $\odot 2020$ by author(s) and Scientific Research Publishing Inc. This work is licensed under the Creative Commons Attribution International License (CC BY 4.0).

http://creativecommons.org/licenses/by/4.0/

\begin{abstract}
Ductal carcinoma in situ (DCIS) is a non-invasive malignancy confined within the basement membrane of the breast ductal system. There is a lot of disparity in the natural history of DCIS with an estimated incidence of progression to invasive ductal carcinoma between $20 \%$ to $53 \%$ over ten or more years after initial diagnosis. The surgical and adjuvant management of DCIS has advanced significantly in the last couple of decades. Nonetheless, surgeons, medical oncologists, and radiation oncologists, along with their patients, still depend on conventional clinical and pathologic risk factors to make management decisions. Irrespective of the management strategy, long-term survival is excellent. The debate around DCIS relates to preventing either under-treatment or over-treatment. In this paper, we will review the incidence and management options of DCIS. Additionally, we will focus on several current disputes related to the management of DCIS, including breast conserving surgery, the role of radiation in breast conservation surgery, sentinel node biopsy in DCIS, hormonal therapy, various risk stratification schemes, and the option of active surveillance for low-risk DCIS.
\end{abstract}

\section{Keywords}

Ductal Carcinoma in Situ, DCIS, Management of DCIS, Treatment of DCIS, Predictors of Recurrence of DCIS, Risk Stratification Schemes for DCIS, Active Surveillance for DCIS

\section{Introduction}

Ductal carcinoma in situ (DCIS) is a malignant intra-ductal proliferation of epithelial cells within the tubular-lobular system of the breast with no microscopic 
evidence of permeation across the basement membrane [1]. There appears to be a progression between flat epithelial atypia, atypical ductal hyperplasia (ADH), and DCIS, in which DCIS is final step prior to the development of invasive disease. The clinical risk factors and molecular aberrations related with malignant transformation are almost indistinguishable between DCIS and invasive cancer [1]. The concurrence of DCIS and invasive carcinoma within one lesion suggests that DCIS is a precursor lesion to invasive carcinoma. Evidence of the ability of DCIS to progress is that $50 \%$ of all recurrences after breast-conserving surgery (BCS) for DCIS, with or without adjuvant treatment, are invasive [2].

Data is sparse on the natural history of DCIS, but some series have reported the outcomes for women many years after undergoing a surgical biopsy that was interpreted as benign that contained an unrecognized area of DCIS [3]. These data identified that approximately $20 \%$ to $53 \%$ of these women developed ipsilateral invasive carcinoma. Sanders et al. [4] reported on 28 women with unrecognized low-grade DCIS in the surgical biopsy specimen, of which 11 developed invasive carcinomas, all of these cancers developed in the same breast and quadrant as the biopsy containing the DCIS (1). The vast majority of these invasive cancers developed within 10 years, but three were diagnosed after 20 years.

Collins et al. [4], in the Nurses' Health Study, singled out 13 women who were found to have DCIS on reexamination of the surgical biopsies that were previously diagnosed as benign. Ten of these women subsequently developed breast cancer; all were ipsilateral, four were DCIS and six were invasive. The interval between the biopsy and the progression to invasive cancer was on average nine years.

Approximately one in eight women (i.e., 12\%) in the United States (US) will be diagnosed with breast cancer in her lifetime, and $20 \%$ to $25 \%$ of these newly diagnosed cases will be DCIS [5]. In 2020, an estimated 48,530 cases of DCIS will be diagnosed in US [5].

Universal screening mammography has resulted in a 10-fold increase in the incidence of DCIS since the mid-1980s, but since 2003, the incidence of DCIS has decreased in women age 50 years and older, conceivably secondary to decline in the use of hormone replacement therapy, while the incidence in women younger than 50 continues to increase [6] [7] [8]. Before the institution of widespread screening mammography in the mid-1980s, most of the cases of DCIS were not identified until a palpable tumor developed, but today, $80 \%$ to $85 \%$ of DCIS cases are screen detected [2] [5].

The incidence of DCIS in necropsy studies is higher than in the general population, proposing that not all DCIS lesions become clinically significant and supporting concerns that most of the increase in DCIS incidence is due to the detection of non-aggressive subtypes that are unlikely to progress to invasive cancer [9].

Most women with DCIS are diagnosed at a median age that ranges from 47 to 63 years, similar to that reported for patients with invasive carcinoma [5]. How- 
ever, the age of peak incidence for DCIS (96.7 per 100,000 women), occurs between the ages of 65 and 69 years, which is younger than that for invasive breast cancer, for which peak incidence (453.1 per 100,000 women), occurs between the ages of 75 and 79 years [5].

The incidence of first-degree relatives having breast cancer (i.e., 10\% to 35\%) as well as deleterious mutations in the breast cancer associated (BRCA) genes are similar for patients with DCIS as for women with invasive breast cancer [10]. Other risk factors for DCIS include: older age, proliferative breast disease, increased breast density, nulliparity, older age at first live birth, history of breast biopsy, early menarche, late menopause, long-term use of postmenopausal hormone replacement therapy, and elevated body mass index in postmenopausal women, are the same as those for invasive breast cancer, but in many cases, the relationship between a given characteristic and invasive cancer is stronger than the relationship between that characteristic and DCIS [11].

\section{Management of DCIS}

The treatment options for DCIS are many including BCS with or without radiation, unilateral mastectomy, bilateral mastectomy, and endocrine therapy with an excellent overall survival (OS) with all (Table 1).

Mastectomy was considered to be the standard of care in the surgical management for all breast cancers, including DCIS. A meta-analysis of eight retrospective studies demonstrated an adjusted 10-year local recurrence rate of $2.6 \%$ ( $95 \%$ confidence interval [CI] $0.8 \%$ to $4.5 \%$ ) after mastectomy, confirming the very low recurrence rate for DCIS treated with mastectomy [12] (Table 2).

In the last decade in the US the use of contralateral prophylactic mastectomy (CPM) in women with unilateral breast cancer has substantially increased, especially in women with DCIS [21] [22]. The risk of developing a contralateral breast cancer in women with DCIS is approximately $\mathbf{0 . 8 \%}$ per year [23]. In spite of the fact that the risk of contralateral breast cancer in DCIS is lower than the risk of ipsilateral recurrence, the cumulative risk of contralateral breast cancer may be clinically relevant because women with DCIS have very low risk of breast cancer mortality from their DCIS.

Randomized clinical trials proved that BCS was equivalent to mastectomy in women with invasive cancer, and this conservative approach has been expanded to include DCIS. There are no randomized prospective trials comparing recurrence rates or overall survival (OS) between mastectomy and BCS for DCIS. Silverstein et al., in a retrospective study, comparing 227 patients with DCIS treated with mastectomy or BCS (with or without RT), showed that the 7-year disease-free survival (DFS) was $98 \%$ for mastectomy and $84 \%$ for BCS, with no difference in OS [24].

The roles of adjuvant RT in women undergoing BCS for DCIS have been studied in four prospective randomized trials [23] [25] [26] [27] (Table 3). Only patients not taking tamoxifen and randomized to radiation or not are included in these four trials. 
Table 1. Treatment options for DCIS.

\begin{tabular}{c}
\hline Treatment Option \\
\hline Breast Conserving Surgery (BCS): \\
- Wide excision alone \\
- Wide excision with radiation (RT) \\
- Wide excision with endocrine therapy \\
Unilateral mastectomy \\
Bilateral mastectomy \\
\hline
\end{tabular}

Table 2. Retrospective studies with an adjusted 10-year local recurrence rate (LRR) of DCIS following mastectomy.

\begin{tabular}{ccc}
\hline Author & \# of Cases & 10-year LRR (\%) \\
\hline Simpson [13] & 30 & 0.0 \\
Wanebo [14] & 14 & 0.0 \\
Millis [15] & 12 & 0.0 \\
Tunon-de-Lara [16] & 74 & 1.4 \\
Owen [17] & 637 & 1.9 \\
Lara [18] & 73 & 4.1 \\
Sunshine [19] & 83 & 4.8 \\
Akashi-Tanaka [20] & 13 & 15.4 \\
Pooled Data & 936 & 3.0 \\
\hline
\end{tabular}

Table 3. Prospective randomized trials evaluating the role of adjuvant RT in women undergoing BCT for DCIS.

\begin{tabular}{ccccccc}
\hline Trial & N & $\begin{array}{c}\text { Median } \\
\text { F/U (yr) }\end{array}$ & $\begin{array}{c}\text { Local } \\
\text { Recurrence NO } \\
\text { RT (\%) }\end{array}$ & $\begin{array}{c}\text { Local } \\
\text { Recurrence } \\
\text { RT (\%) }\end{array}$ & $\begin{array}{c}\text { Risk } \\
\text { Reduction } \\
(\%)\end{array}$ & p Value \\
\hline NSABP B-17 [23] [28] & 818 & 17.25 & 35 & 20 & $47-52$ & $\mathrm{p}<0.001$ \\
EORTC 10853 [26] & 1010 & 15.8 & 31 & 18 & 48 & $\mathrm{p}<0.001$ \\
UK/ANZ DCIS [25] & 475 & 12.7 & 25 & 9 & 69 & $\mathrm{p}<0.0001$ \\
SweDCIS [27] & 1046 & 17 & 27 & 12 & 37.5 & $\mathrm{p}<0.001$ \\
\hline
\end{tabular}

The NSABP B-17 was the first phase III randomized study that compared lumpectomy alone to lumpectomy plus breast radiation in 818 patients with localized DCIS [23] [28]. In the BCS only group $(\mathrm{N}=391)$ the event free survival was $\mathbf{7 3 . 8 \%}$ and in the BCS plus RT group $(\mathrm{N}=399)$ the event free survival $\mathbf{8 4 . 4 \%}$. In the BCS only group $(\mathrm{N}=391)$ the local recurrence rate was $35 \%$ and in the BCS plus RT group $(\mathrm{N}=399)$ local recurrence rate was $20 \%$. The trial concluded that RT significantly decreases the rate of invasive cancer and DCIS in the ipsilateral breast by approximately $\mathbf{5 0 \%}$ [28]. In a recent update of the NSABP B-17 with 15 year follow-up, the invasive ipsilateral breast tumor recurrence (I-IBTR) for the BCS only group was $\mathbf{1 9 . 4 \%}$ compared to the BCS plus RT group of $\mathbf{8 . 9 \%}$ and they also found that there is an increase rate of breast cancer related mortality with I-IBTR but not with DCIS [23]. 
The Swedish breast cancer cooperative group (BCCG) accrued 1046 patients between 1987 and 1999 with a mean follow-up of eight years [27]. Inclusion criteria included a clinically negative axilla, localized DCIS to less than one quadrant of the breast. In the BCS only group $(\mathrm{N}=520)$ the local recurrence rate was $27 \%$ and in the BCS plus RT group $(\mathrm{N}=526)$ local recurrence rate was $12 \%$. The study identified that there was an increasing effect of RT with age, with patients under 50 years having a $6 \%$ absolute reduction of local recurrence with RT compared to an $18 \%$ absolute reduction of local recurrence with RT in patients older than 65 years [27].

The European Organization for Research and Treatment of Cancer (EORTC) 10853 accrued 1010 patients between 1986 and 1996 [26]. Inclusion criteria included patients younger than 70 years, completely excised DCIS measuring equal or less than $5 \mathrm{~cm}$. In the BCS only group $(\mathrm{N}=503)$ the local recurrence rate was $31 \%$ and in the BCS plus RT group $(\mathrm{N}=507)$ local recurrence rate was $18 \%$ [26]. The EORTC 10853 found similar findings to the NSABP B-17 study in that I-IBTR was associated with a decrease in breast cancer specific survival (BCSS) but not with DCIS [23] [26].

The Early Breast Cancer Trialists' Collaborative Group (EBCTCG) carried out an individual patient-level meta-analysis of these four randomized controlled trials, which included 3729 women [29]. The use of RT after BCS decreased the risk of recurrence by approximately $50 \%$ (hazard ratio [HR] $0.49 ; 95 \%$ CI, 0.41 to 0.58 ; $\mathrm{p} \leq 0.00001$ ), equivalent to an absolute 10 -year reduction in the risk of any ipsilateral breast recurrence of $\mathbf{1 5 . 2 \%}$ ( $28.1 \%$ [no RT] versus $12.9 \%$ [RT]; $\mathrm{p} \leq 0.00001$ ) [29]. In the EBCTCG study, the number needed to treat with RT was nine women to prevent one ipsilateral breast recurrence. After 10 years of follow-up, there was no notable effect on breast cancer mortality, mortality from causes other than breast cancer, or all-cause mortality. In all subgroup of patient, including those with small, low-grade tumors and negative margins, RT decreased the risk of an ipsilateral breast event by approximately 50\% [29]. Hence, though RT clearly reduces local recurrence rates for women undergoing BCS for DCIS, it does not improve survival. In view of the fact that breast RT has rare but potentially serious side effects (RT-induced malignancy and cardiovascular disease) [30], its risks should be weighed against the decrease in local recurrence when making treatment recommendations for patients with DCIS (Table 4).

Two randomized clinical trials have assessed the role of tamoxifen in preventing recurrence (Table 5) [26] [31]. The NSABP B-24 was a double-blind prospective trial of adjuvant tamoxifen ( $20 \mathrm{mg}$ /day) versus placebo for five-years in 1,804 women undergoing BCS and adjuvant RT [31]. This studied accrued patients between 1991 and 1994. Roughly 25\% had positive or uncertain margins, and 80\% had lesions measuring $1 \mathrm{~cm}$ or less. The 15-year cumulative incidence of recurrence was reduced from $18.3 \%$ to $16.0 \%$ with the addition of tamoxifen, with a reduction in invasive recurrence from $10 \%$ to $8.5 \%$. The inclusion of tamoxifen also had a chemopreventive effect on the contralateral breast, with the 15 -year cumulative incidence of all contralateral breast cancers (CBC) reduced from $\mathbf{1 0 . 8 \%}$ to $7.3 \%$. The use of tamoxifen did not improve overall survival (OS) [31]. 
Table 4. Breast cancer mortality in randomized radiation therapy (RT) trials for women with DCIS treated with BCS.

\begin{tabular}{ccccccc}
\hline Trial & N & $\begin{array}{c}\text { Median } \\
\text { F/U (yr) }\end{array}$ & $\begin{array}{c}\text { Breast Cancer } \\
\text { Mortality NO RT (\%) }\end{array}$ & $\begin{array}{c}\text { Breast Cancer } \\
\text { Mortality RT (\%) }\end{array}$ & RR & p Value \\
\hline NSABP B-17 [23] [28] & 818 & 17.25 & 3.1 & 4.7 & 1.44 & NS \\
EORTC 10853 [26] & 1010 & 15.8 & 5.0 & 4.0 & 0.80 & NS \\
UK/ANZ DCIS [25] & 811 & 12.7 & 2.0 & 1.5 & 0.74 & NS \\
SweDCIS [27] & 1046 & 17 & 4.2 & 4.1 & 0.98 & NS \\
\hline
\end{tabular}

Table 5. Prospective randomized trials of adjuvant tamoxifen for women with DCIS undergoing BCS.

\begin{tabular}{|c|c|c|c|c|c|c|c|c|}
\hline Trial & RT & Placebo & $\mathbf{N}$ & $\begin{array}{c}\text { Median F/U } \\
(y r)\end{array}$ & $\begin{array}{c}\text { Local Recurrence } \\
\text { Rate (\%) }\end{array}$ & $\begin{array}{l}\text { Contralateral Breast } \\
\text { Cancer Rate (\%) }\end{array}$ & $\begin{array}{l}\text { Breast Cancer } \\
\text { Mortality (\%) }\end{array}$ & $\begin{array}{c}\text { Overall } \\
\text { Death (\%) }\end{array}$ \\
\hline \multirow[t]{2}{*}{ NSABP B-24 [31] } & $\mathrm{RT}$ & Placebo & 900 & 13.6 & 16.6 & 8.1 & 2.7 & 14.4 \\
\hline & & Tamoxifen & 899 & 13.6 & 13.2 & 4.9 & 2.3 & 12.2 \\
\hline \multirow[t]{4}{*}{ UK/ANZ DCIS [26] } & No RT & Placebo & 544 & 12.7 & 11.6 & 2.8 & 2.0 & 10 \\
\hline & & Tamoxifen & 567 & 12.7 & 8.7 & 1.4 & 3.0 & 10 \\
\hline & $\mathrm{RT}$ & Placebo & 267 & 12.7 & 4.0 & 1.7 & 1.0 & 10 \\
\hline & & Tamoxifen & 316 & 12.7 & 3.4 & 1.5 & 2.0 & 14 \\
\hline
\end{tabular}

A $2 \times 2$ factorial randomized trial of both $\mathrm{RT}$ and tamoxifen by the United Kingdom, Australia, and New Zealand (UK/ANZ) DCIS group accrued patients between 1990 to 1998 [26]. The study allowed women to participate in either of the randomization arms (19). A total of 1576 women participated in the tamoxifen randomization, of whom 1053 did not receive RT and 523 did. The use of tamoxifen decreased the incidence of ipsilateral DCIS recurrence (HR 0.70; 95\% CI, 0.51 to $0.86 ; \mathrm{p}=0.03$ ) but had no effect on ipsilateral invasive disease (HR 0.95 ; $95 \% \mathrm{CI}, 0.66$ to $1.38 ; \mathrm{p}=0.8$ ). The ipsilateral benefit of tamoxifen was limited to those not receiving RT (HR for ipsilateral breast events $0.77 ; 95 \% \mathrm{CI}$, 0.59 to $0.98 ; \mathrm{p}=0.04$ ) and was not significant in those receiving $\mathrm{RT}$. The radiation analysis of the study is shown in Table 6. The chemopreventive effect of hormonal therapy was shown with a decrease in the incidence of any $\mathrm{CBC}$ events (HR 0.44; 95\% CI, 0.25 to $0.77 ; \mathrm{p}=0.005$ ) [26].

As with the NSABP B-24, tamoxifen resulted in no survival advantage. Patients were enrolled in the NSABP B-24 and UK/ANZ trials without knowledge of hormone receptor status. Allred et al. tested a subset of 732 cases (41\%) from NSABP B-24 for estrogen receptors. They identified that the benefit of tamoxifen was limited to the ER-positive group [31].

In the non-randomized Eastern Oncology Cooperative Group and Cancer Therapy and the American College of Radiology Imaging Network (ECOG ACRIN) E5194 trial, women with DCIS with low-risk clinical and pathologic characteristics were assigned to two cohorts between April 1997 and October 2002: cohort one ( $\mathrm{n}=$ 561 ) had low- (50\%) or intermediate-grade (50\%) DCIS and a tumor size $\leq 2.5 \mathrm{~cm}$; and cohort two $(\mathrm{n}=104)$ had high-grade DCIS and a tumor size $\leq 1 \mathrm{~cm} \mathrm{[32].}$ 
Table 6. Radiation analysis of the UK/ANZ DCIS trial [26].

\begin{tabular}{cccc}
\hline & Radiation & No Radiation & p-value \\
\hline Ipsilateral & & & \\
- Invasive & $3 \%$ & $6 \%$ & 0.01 \\
- DCIS & $3 \%$ & $7 \%$ & 0.0004 \\
Contralateral & Not affected by radiation & Not affected by radiation & Not affected by radiation \\
Total DCIS & $3 \%$ & $9 \%$ & $<0.0001$ \\
Total Invasive & $5 \%$ & $7 \%$ & 0.07 \\
\hline
\end{tabular}

Enrollment and cohort assignment were based on clinical and pathology assessment at treating institutions. All patients underwent surgical excision (lumpectomy) of the primary DCIS tumor and received no radiation therapy. Lumpectomy was to have a minimum negative margin width of $\geq 3 \mathrm{~mm}$ or no tumor on re-excision. The primary endpoint of the study was the development of an ipsilateral breast event, defined as the first local recurrence of DCIS or invasive carcinoma in the treated breast. Tamoxifen was not randomly assigned. The median follow-up of the trial was 12.3 years. A total of 99 ipsilateral breast events were observed (74 in cohort 1 and 25 in cohort 2), including 51 invasive ipsilateral breast events (52\%; 39 in cohort 1 and 12 in cohort 2) [32].

Overall the IBTR rate in the low to intermediate grade DCIS group was $14.4 \%$ compared to the high-grade DCIS group which was $26.4 \%(\mathrm{p}=0.003$, HR 1.84). The I-IBTR rate in the low to intermediate grade DCIS group was $7.5 \%$ compared to the high-grade DCIS group which was $13.4 \%(\mathrm{p}=0.08)$. The risks of developing an ipsilateral breast event and an invasive event increased over time through 12 years, with no plateau observed. In group one, the rates of developing an ipsilateral breast event and an invasive ipsilateral breast event were approximately $1.2 \%$ and $0.6 \%$ per year through year the 12 years. At 12 years, overall survival was $84.0 \%$ and $82.8 \%(\mathrm{p}=0.96)$ and the incidence of contralateral breast cancer events was $6.7 \%$ and $12.0 \%(\mathrm{p}=0.16)$. On multivariable analysis, study cohort (hazard ratio $[\mathrm{HR}]=1.84, \mathrm{p}=0.009$, for cohort 2 vs 1 ) and tumor size $(\mathrm{HR}=2.11, \mathrm{p}=0.03$, for $>10 \mathrm{~mm}$ vs $\leq 5 \mathrm{~mm})$ were significantly associated with de development of an ipsilateral breast event. On multivariable analysis for invasive ipsilateral breast event, cohort was of borderline significance $(p=0.08)$ [32].

The conclusion of the E5194 trial was that for patients with DCIS of the breast who had favorable clinical and pathologic factors and were managed with surgical excision alone (without radiation) the study demonstrated that the risks of developing an ipsilateral breast event and an invasive ipsilateral breast event increased over time through 12 years of follow-up, without plateau. Individual patients and their physicians will need to decide if these 12-year risks are acceptable, and to judge whether or not to add adjuvant treatment after surgical excision [32].

The Radiation Therapy and Oncology Group (RTOG) 9804 trial was a prospective randomized trial that accrued 636 patients from 1998 to 2006 with mam- 
mographically detected low-grade or intermediate-grade DCIS, measuring less than $2.5 \mathrm{~cm}$ with margins $\geq 3 \mathrm{~mm}$, and compared RT (with or without tamoxifen) with observation (with or without tamoxifen) after surgery [33]. The trial was designed for 1790 patients but was closed early because of lower than projected accrual. Ipsilateral local failure (LF) was the primary end point. Tamoxifen was optional if started within four weeks of DCIS diagnosis (62\% of the patients did receive tamoxifen). Table 7 demonstrates the LF rate based on histologic grade and tamoxifen use. With a median follow-up time was 7.17 years, two LFs occurred in the RT arm (with or without tamoxifen), and 19 LFs occurred in the observation arm (with or without tamoxifen). At seven years, the LF rate was $0.9 \%$ (95\% CI, $0.0 \%$ to $2.2 \%$ ) in the RT arm (with or without tamoxifen) compared to $6.7 \%$ (95\% CI, 3.2\% to 9.6\%) in the observation arm (with or without tamoxifen) (hazard ratio, $0.11 ; 95 \% \mathrm{CI}, 0.03$ to $0.47 ; \mathrm{p} \leq 0.001$ ). The conclusion of the trial said that the good-risk subset of patients with DCIS, the LF rate was low with observation but was decreased significantly with the addition of RT [33].

The differences in the rates of local recurrence, in the patients who did not receive RT, between the NSABP B-24 (20.9\%) and the RTOG 9804 (6.7\%) can be attributed to the fact that the B-24 trial did not separate patients in low-, intermediate-, or high-grade groups [31] [33]. The differences in recurrence rate between the ECOG ACRIN E5194 (10.5\%) and the RTOG 9804 (6.7\%) trials in the low risk groups may be secondary to the higher proportion of patients who took tamoxifen in the RTOG 9804 trial [32] [33].

Two trials, the NSABP B-35 [34] and International Breast Cancer Intervention Studies (IBIS)-II [35], compared anastrozole (an aromatase inhibitor proven superior to tamoxifen for invasive cancer) with tamoxifen in hormone receptor-positive DCIS managed with local excision [34] [35]. Both studies were randomized and double-blinded. The NSABP B-35 was a phase III clinical trial that randomized postmenopausal women with ER-positive DCIS to five years of anastrozole or tamoxifen following BCS and whole breast RT. The trial sought to determine how effective anastrozole is compared to tamoxifen in preventing a breast cancer recurrence, as well as the quality of life of patients taking anastrozole. It accrued 3104 women between 2003 and 2006 with nine years of follow up [34]. The inclusion criteria included postmenopausal women with estrogen and progesterone receptor (ER/PR) positive DCIS and/or LCIS, that were excised with negative margins.

Table 7. Local failure by tamoxifen use and histological grade [33].

\begin{tabular}{ccc}
\hline Tamoxifen & Histologic Grade & Local Failure Rate (\%) \\
\hline Yes & Low & 1.2 \\
Yes & Intermediate & 2.0 \\
No & Low & 5.3 \\
No & Intermediate & 8.4 \\
\hline
\end{tabular}


Investigators found significantly fewer breast cancer events in the anastrozole group $(n=90)$ compared to the tamoxifen group $(n=122)(H R, 0.73$; confidence interval, $0.56-0.96, \mathrm{p}=0.0234)$. The estimated 10 -year breast-cancer-free interval rates were $\mathbf{9 3 . 5 \%}$ for the anastrozole group compared to $\mathbf{8 9 . 2 \%}$ for the tamoxifen group. This recorded difference in breast cancer-free interval was attributable almost entirely to younger postmenopausal women less than 60 years of age. Interestingly the difference between treatments did not become apparent until after five years of follow-up, likely due to the low number of events in both groups. There was no difference in OS between the two treatment groups [34].

The IBIS-II accrued 2980 women between 2003 to 2013 from 238 centers in 17 countries with a follow-up of seven years. The study randomized postmenopausal women with ER positive DCIS (micro-invasion was permitted), negative surgical margins, and adjuvant RT according to local practices (70\% of patients received $\mathrm{RT}$ ) to lumpectomy plus anastrazole to lumpectomy plus tamoxifen. The incidence of any breast cancer event was low in both groups (5\%), and anastrozole was non-inferior to tamoxifen [35].

DCIS is a non-invasive entity and lymph node involvement is not expected to occur. If a patient presents with DCIS in the breast but is found to have malignant cells in the lymph nodes preoperatively, the patient has, by definition, invasive breast cancer and not DCIS. In situations where patients have large tumors (greater than $4 \mathrm{~cm}$ ) or extensive micro-calcifications, a focus of invasion can be missed because of limited pathologic sampling (there is roughly a $20 \%$ to $30 \%$ rate of associated invasive disease reported on final pathology in patients who were diagnosed with DCIS on stereotactic biopsy) [36]. These patients, as well as those with high-grade or palpable disease, are at higher risk for lymph node involvement and may also warrant sentinel lymph node (SLN) mapping, particularly if they are undergoing mastectomy or if BCS is to be followed by oncoplastic surgery. The risk of not identifying metastatic disease in the lymph nodes must be weighed against the risk of lymphedema associated with SLN dissection in each patient.

Cox et al., in 1998 [36], reported a positive SLN biopsy rate of 6\% in patients with DCIS when a combination of hematoxylin-eosin staining and immunohistochemistry was utilized. Another study by et al. [37] identified a positive SLN biopsy rate of $12 \%$ in patients with DCIS considered to be at high risk for invasion and among $10 \%$ of patients who had DCIS with micro-invasion. Invasive carcinoma is identified when DCIS is diagnosed by core needle biopsy of a tumor mass in $25 \%$ of the cases compared to $13 \%$ of the cases when the biopsy is performed for micro-calcifications. The incidence of identifying a positive SLN is more frequent in large areas of DCIS (odds ratio of 1.14 per $1 \mathrm{~cm}$ increase in size), when comedo necrosis is present (up to $40 \%$ of the cases), when the DCIS is high nuclear grade ( $23 \%$ vs $7 \%$ low-intermediate grade), and when estrogen receptors (ER)/progesterone receptors (PR) are negative (33\% vs $11 \%$ for $\mathrm{ER} / \mathrm{PR}$ positive disease) [37].

A study from the MD Anderson Cancer Center (MDACC) [38], investigated 
the incidence of positive lymph nodes and the clinical significance of these findings in 1234 patients with an initial diagnosis of DCIS planned for SLN dissection. The positive SLN biopsy rate was $10.7 \%$ (positive SLNs in 132 patients). The results included isolated tumor cells in $66(5.4 \%)$ patients, micro-metastases in $36(2.9 \%)$ and macro-metastases in $30(2.4 \%)$. There was upstaging in the primary tumor in $26.5 \%$ of the cases ( 327 patients) to micro-invasion or invasive cancer. Factors predictive of a positive SLN were diagnosis by excisional biopsy, papillary histology, DCIS greater than $2 \mathrm{~cm}$, more than three interventions prior to SLN dissection (needle biopsy, excisional biopsy, etc.), micro-invasion or invasive cancer. It was noted that with increasing numbers of preoperative interventions (biopsy, needle localization, etc.), there was a higher likelihood of positive SLN findings with the majority being isolated tumor cells $(44 / 907,4.2 \%)$ or micro-metastases $(12 / 907,1.3 \%)$. There were no macro-metastases identified in the SLNs of patients with pure DCIS. The high incidence of isolated tumor cells in the SLNs with increasing preoperative interventions supports the concept of benign mechanical transport of epithelial cells during manipulation of the primary tumor. At a median follow-up of 61.7 months, there was no difference in disease-free survival (DFS) outcomes in the patients with pure DCIS with or without positive SLN findings [38].

In general terms, SLN dissection should be limited to patients who undergo mastectomy for large, high-grade DCIS because it is difficult to perform lymphatic mapping after a mastectomy if invasive cancer is found in the mastectomy specimen. The 2017 ASCO guidelines do not recommend SLN biopsy for BCS for DCIS but for large, high-grade DCIS with necrosis one can discuss with the patient the option of performing the SLN biopsy during BCS [39].

\section{Observation Only for Low-Risk DCIS}

Recognizing the need for stronger evidence to help drive the management changes of DCIS, many groups around the world have developed randomized, prospective clinical trials studying operative vs. non-operative treatment of low-risk DCIS. The rationale for these studies is that not all DCIS cases are clinically and biologically the same, but up to now most forms of the disease are treated in a similar, uniform fashion (with an operation) based on retrospective data.

The Comparison of Operative versus Monitoring and Endocrine Therapy (COMET) trial is a phase III, randomized controlled study for patients with low-risk DCIS [40]. The primary outcome is ipsilateral invasive breast cancer rate in women undergoing guideline concordant care (GCC) compared with active surveillance (AS). Secondary endpoints will be to compare surgical, oncological and patient-reported outcomes. Patients randomized to the GCC group will undergo surgery as well as radiotherapy when appropriate; those in the AS group will be monitored closely with surgery only on identification of invasive breast cancer. Patients in both the GCC group and AS group will have the option of endocrine therapy. The total planned accrual goal is 1200 patients. Table 8 and Table 9 show the inclusion and exclusion criteria of the COMET trial [40]. 
Table 8. Inclusion criteria of the COMET Trial [40].

- New diagnosis of DCIS without invasive breast cancer

- Unilateral, bilateral, unifocal, or multifocal DCIS

- A patient who has had a lumpectomy with positive margins as part of their treatment for a current DCIS diagnosis is eligible

- No previous history of breast cancer (DCIS or invasive cancer) in either breast prior to current DCIS diagnosis

- 40 years of age or older at the time of DCIS diagnosis

- ECOG performance status 0 or 1

- No contraindication for surgery

- Baseline imaging:

○ Unilateral DCIS:

- Contralateral normal mammogram $\leq 6$ months of registration and ipsilateral breast imaging $\leq 120$ days of registration

- Bilateral DCIS:

- Bilateral breast imaging $\leq 120$ days of registration

- Pathological criteria:

- ADH suspicious for DCIS

- Any grade I or grade II DCIS

Absence of invasive or microinvasive breast cancer

- Diagnosis confirmed on core needle, vacuum-assisted biopsy or surgery $\leq 120$ days of registration

○ $\mathrm{ER}(+)$ and/or PR(+) by IHC ( $\geq 10 \%$ staining or Allred score $\geq 4)$

- HER2 $0,1+$ or $2+$ by IHC if HER2 testing is performed

- Histology slides reviewed and agreement between two clinical pathologists that pathology fulfills COMET eligibility criteria

- At least two sites of biopsy for those cases where mammographic extent of calcifications exceeds $4 \mathrm{~cm}$, with second biopsy benign or both sites fulfilling pathology eligibility criteria

- Amenable to follow-up examinations

Ability to read, understand and evaluate study materials and willingness to sign a written informed consent document in Spanish or English

Table 9. Exclusion criteria of the COMET trial [40].

$\circ$ All grade III DCIS

- Male DCIS

- Concurrent diagnosis of invasive or micro-invasive breast cancer in either breast prior to randomization

- Documented mass on examination or imaging at the site of DCIS prior to biopsy yielding diagnosis of DCIS

- Bloody nipple discharge or skin changes associated with DCIS

- Mammographic finding of BI-RADS 4 or greater within 6 months of registration at site other than that of known DCIS, without pathological assessment

- Use of investigational cancer agents within 6 weeks prior to diagnosis

- Any serious and/or unstable pre-existing medical, psychiatric or other existing condition that would prevent compliance with the trial or consent process

- Pregnancy

- Documented history of prior tamoxifen, aromatase inhibitor, or raloxifene in last 6 months

Patients randomized to the GCC arm will undergo appropriate surgery for DCIS according to local guidelines. It is expected that patients will complete definitive surgery within 60 days of randomization. Data on all related surgical procedures, including data on immediate or delayed breast reconstruction, will be collected. 
If a patient is randomized to the GCC arm opts for AS, they will be considered as a "crossover" and will continue to participate in completion of patient-reported outcome surveys. The recommendation for adjuvant RT should be decided following surgery and recommended according to standard local protocols. The use of adjuvant RT is not mandated within the trial. However, data pertaining to the use of RT will be collected. The use of endocrine therapy is not mandatory, but patients are encouraged to discuss this with their providers in both arms of the trial. Selection of endocrine therapy will be determined based on provider recommendation and patient preference and administered for a maximum duration of five years. If applicable, data regarding the use of endocrine therapy (type, duration, adherence and side effects) will be captured at each visit and patient-reported adherence will be measured in follow-up surveys [40].

Patients in the AS arm will not undergo surgery unless a biopsy during surveillance documents invasive breast disease which requires surgical intervention. If the patient opts for surgery in the absence of invasive breast cancer, they will be considered as a "crossover" and will continue to participate in completion of patient-reported outcome surveys. For both the GCC and AS groups, required surveillance consists of clinical examination, including history and physical examination, every six months for a minimum of five years and every 12 months thereafter, up to seven years from the time of registration. Patients on the GCC arm who have not had a mastectomy will have bilateral mammography annually; those on the AS arm will have ipsilateral mammography every six months and contralateral mammography every 12 months. Clinical criteria requiring further investigation include new breast signs and symptoms such as new breast tumor; nipple/skin retraction; nipple discharge and breast edema/erythema on clinical examination in either breast. Radiographic criteria for biopsy include an increase in extent of calcifications $\geq 5 \mathrm{~mm}$ in at least one dimension compared with the most recent prior mammogram in the index breast as well as new suspicious findings on other radiological studies (US, MRI) in either breast.

The strengths of the COMET trial are that it is a randomized controlled clinical trial, follows patients who crossover form the AS arm to GCC (surgery) or those who are randomized to surgery but elect AS. The study continues to collect data on women who refuse randomization to help study selection bias and make the trial more generalizable. Multiple secondary and addition endpoints are included in the studied (OS, number of radiologic studies, assessment of anxiety, depression, and tolerance of uncertainty. The weaknesses of the trial include variation of pathologic diagnosis of low-grade DCIS and the comparator arms are very different from one another [40].

The Low Risk DCIS trial (LORIS) is a multi-center, randomized (1:1), controlled phase III trial of surgery versus active monitoring in patients with low risk DCIS, incorporating a two-year internal feasibility study [41]. The trial was initiated in 2014 and the goal is to accrual 932 women with confirmed low risk DCIS. This study is based on a non-inferiority margin defined as an absolute reduction in 
the five-year ipsilateral invasive breast cancer free survival rate at five years of $2.5 \%$ (i.e. from $97.5 \%$ to $95 \%$ ). Table 10 and Table 11 show the inclusion and exclusion criteria of the LORIS trial [41].

Trial assessments in the surgery arm includes annual mammography for a minimum of ten years, patient reported outcomes (QoL and Health Economics) for five years, and collection of follow-up data via annual follow-up appointment for years one to five and via annual telephone call to patient for years six to ten. Trial assessments in the active monitoring arm include annual mammography for a minimum of ten years, patient reported outcomes (QoL and Health Economics) for five years and collection of follow-up data via annual telephone call to patient for ten years [41].

Table 10. Inclusion criteria of the LORIS trial [41].

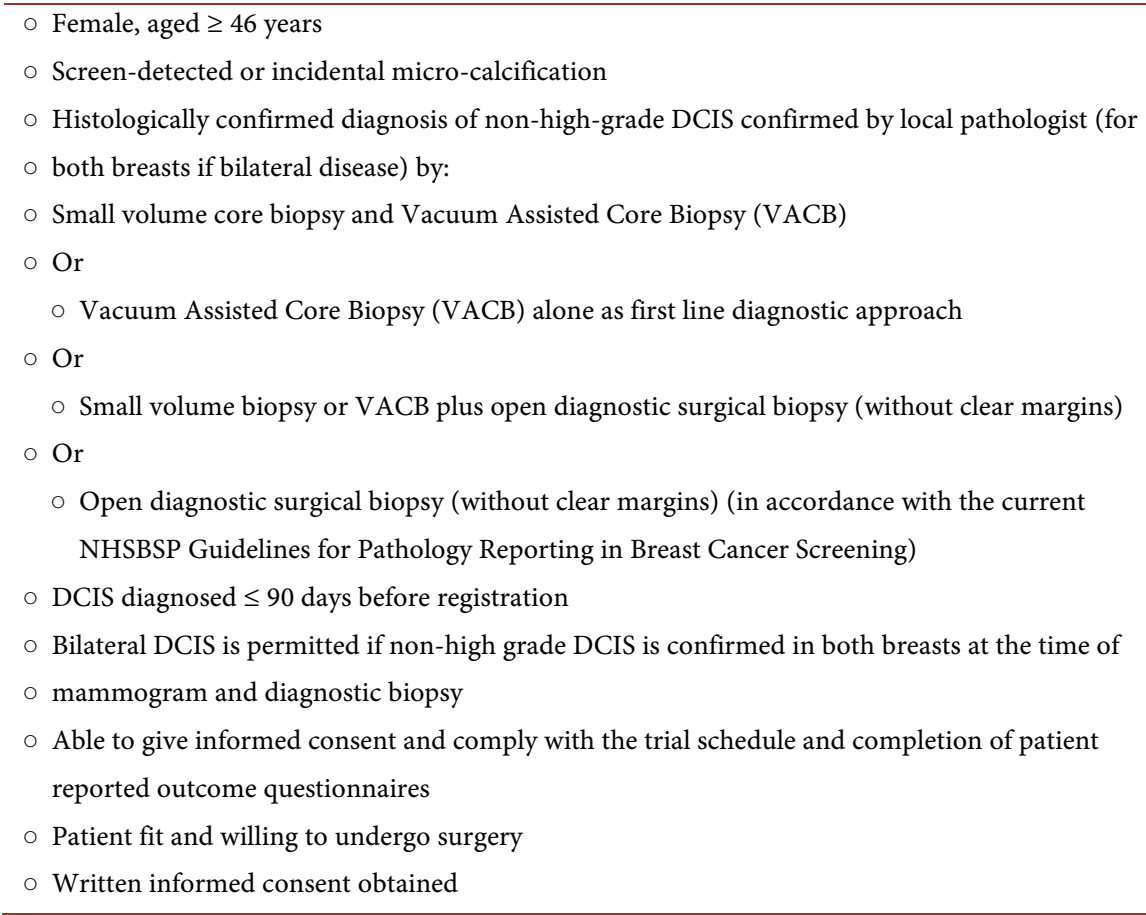

Table 11. Exclusion criteria for the LORIS trial [41].

\footnotetext{
- Previous or current diagnosis of invasive breast cancer or previous ipsilateral DCIS (previous surgically treated contralateral DCIS is permitted)

- A mass lesion clinically on imaging at the site of the micro-calcification which has not been proven on biopsy to be a specific benign lesion

- Surgical procedure with curative intent (even if clear margins have not been achieved)

- Unequivocal comedo necrosis observed

- Any serious and/or unstable pre-existing condition that would prevent compliance with the trial or the consent process

- Recent onset ipsilateral blood-stained nipple discharge without benign explanation

○ High risk group for developing breast cancer (as defined in current NICE guidelines for familial breast cancer, or due to prior exposure to mantle field radiotherapy
} 
The LORD (Low Risk DCIS) trial is a randomized, international, multi-center, phase III non-inferiority trial, that aims to determine whether screen-detected low-risk DCIS can safely be managed by an active surveillance strategy or that the conventional treatment, being either wide local excision (WLE) alone, WLE + RT, or mastectomy, and possibly hormonal therapy (HT), should remain the standard of care. The primary endpoint is ipsilateral invasive breast cancer-free rate at ten years [42]. The study started accruing in 2017 and the estimated enrollment is 1240 women. Table 12 and Table 13 show the inclusion criteria for the LORD trial.

Table 12. Inclusion criteria for the LORD trial [42].

- Women of age $>$ or $=45$ years

- Any menopausal status

- Calcifications only lesions, detected by population-based or opportunistic screening mammography

- Within twelve weeks of detection at least six $12 \mathrm{G}$ biopsies (or the equivalent of six $12 \mathrm{G}$ needles) need to be taken from the area of the calcification:

- This implies at least $7 \mathrm{cc}$ volume irrespective of supplier, or at least three $8 \mathrm{G}$, four $9 \mathrm{G}$, five $10 \mathrm{G}$ or five $11 \mathrm{G}$ biopsy needles need to be taken to meet LORD's eligibility criteria

- Whatever needle size is applied, it is essential to confirm that the biopsies contain representative calcifications via biopsy radiography, microscopy, or both

- Any size DCIS

- Marker placement at biopsy site(s) in the breast

- Good correlation between pathological and radiological findings i.e. both findings confirm low-risk DCIS and no suspicion of high-grade DCIS or invasive breast cancer

- Prior surgery of the ipsilateral breast because of a benign lesion allowed

- ASA score 1 or 2

- Before patient registration/randomization, written informed consent must be given according to $\mathrm{ICH} / \mathrm{GCP}$, and national/local regulations

Table 13. Exclusion criteria for the LORD trial [42].

○ Presence of mass, increased density around calcifications, architectural distortion, stellate lesion on mammography

- Presence of bilateral DCIS

- Presence of Paget's disease, invasive breast cancer, or pleomorphic LCIS

- Presence of symptomatic DCIS e.g. DCIS detected by palpation or nipple discharge

- Presence of synchronous invasive carcinoma in the contralateral breast

- Prior history of invasive breast cancer or DCIS

- Prior history of other cancer except carcinoma in situ of the cervix or basocellular carcinoma of the skin

○ Presence of serious disease that precludes definitive surgical treatment (e.g. cardiovascular/pulmonary/renal disease

o Individual with a family member with a known gene mutation associated with increased risk of breast cancer, unless study participant is a proven non-carrier of mutation

- Presence of pregnancy or breast-feeding

- Presence of any psychological, familial, sociological or geographical condition potentially hampering compliance with the study protocol and follow-up schedule; those conditions should be discussed with the patient before registration in the trial 
In a study by Pilewskie et al. [43], reviewed Memorial Sloan Kettering Cancer Center (MSKCC) long-term results of patients with DCIS that would or would not meet the "low-risk" criteria as defined by the LORIS trial. All patients were treated with standard surgical excision \pm adjuvant radiation therapy. Therefore, their "low-risk" population by design is at lower risk than those in the LORIS trial, because all of their patients underwent complete surgical excision of the index DCIS and neither a high-grade nor an invasive component was identified. They identified 2394 women of which 401 met the LORIS criteria with a median follow-up of 5.9 yrs ( $431 \mathrm{had} \geq 10$ years follow-up). The LORIS cohort median age was 61 years (range: 46 to 86); 207 (52\%) underwent RT, 79 (20\%) received endocrine therapy, 24 of the 401 experienced an IBTR. Overall 10-year IBTR rates were $10.3 \%$ (LORIS) versus $15.4 \%$ (non-LORIS) $(\mathrm{p}=0.08)$ and without $\mathrm{RT}, 12.1 \%$ versus $21.4 \%$, respectively $(\mathrm{p}=0.06)$. The 10 -year invasive-IBTR rates for women meeting LORIS criteria were $5.3 \%$ BCS overall and $6.0 \%$ without RT. The authors concluded that women meeting LORIS criteria (after complete surgical excision) are at somewhat lower risk for IBTR. Among such women undergoing excision without RT, the 10-year invasive-IBTR rate was $6 \%$. Given approximately $20 \%$ of women with core-biopsy-proven non-high-grade DCIS have invasive cancer at excision, women managed without excision would be expected to experience higher invasive cancer rates. Additional criteria are needed to identify women not requiring intervention for DCIS [43] [44].

The study leaders from the LORIS, LORD, COMET, and LORRETA trials have regular meetings as well as similar structure, data and surveys to foster collaboration with the future goal of combining data/analysis and for consensus building [44]. Table 14 is a summary of all studies evaluating AS for low-grade DCIS.

Table 14. Summary of all studies evaluating AS vs standard of care for low-grade DCIS [44].

\begin{tabular}{ccccc}
\hline & LORIS & LORD & COMET & LORRETA \\
\hline Phase & III & III & III & III \\
Study & AS & AS & AS + ET & AS + ET \\
Screening & MMG & MMG & MMG & MMG, US, MRI \\
Nuclear Grade & 1 or 2 & 1 & 1 or 2 & 1 or 2 \\
Comedo Necrosis & No & No & Eligible & No \\
ER & N/S & N/S & Positive & Positive \\
HER2 & N/S & N/S & Negative & Negative \\
Size & N/S & Any Size & Any Size & Equal or less $2.5 \mathrm{~cm}$ \\
Patient/Target & $113 / 932$ & $25 / 1240$ & $182 / 1200$ & $27 / 340$ \\
\hline
\end{tabular}




\section{Predictors of Recurrence}

Multiple features of DCIS are related with a less favorable clinical outcome. Pathologic factors, such as large tumor size (greater than $3 \mathrm{~cm}$ ), high nuclear grade, comedo necrosis, and positive margins, are associated with a greater risk of local recurrence [45]. Positive surgical margins are the most important independent prognostic variable for predicting local recurrence.

Lagios et al. in 1989 [46] recognized high nuclear grade and comedo necrosis as factors predictive of local recurrence. Patients whose tumors had a high nuclear grade and comedo necrosis had a $19 \%$ local recurrence rate after BCS alone at a mean interval of 26 months, compared with $\mathbf{5 \%}$ for those patients whose tumors were low-nuclear grade and did not have comedo necrosis. Silverstein et al. in 1995 [47], developed the Van Nuys algorithm to stratify patients into three risk groups utilizing three established predictors of local recurrence: tumor size, margin width, and pathologic classification (the presence or absence of comedo necrosis and/or high tumor grade).

Updated to include age, the University of Southern California/Van Nuys Prognostic Index Scoring System (USC/VNPI) can be used to determine which patients with DCIS are at greatest risk for recurrence and would therefore benefit from particular forms of local or regional therapy [48]. With the goal being a local recurrence rate of less than $20 \%$ at 12 years, BCS alone is recommended for scores 4 to 6 , and for patients with a score of 7 but have margins $\geq 3 \mathrm{~mm}$. BCS plus RT is recommended for patients with a score of 7 and margins $<3 \mathrm{~mm}$, a score of 8 and margins $\geq 3 \mathrm{~mm}$, or a score of 9 and margins $\geq 5 \mathrm{~mm}$. Mastectomy is recommended for a score of 8 and margins $<3 \mathrm{~mm}$; a score of 9 and margins $<5 \mathrm{~mm}$; and for all patients with a score of 10 to 12 . The USC/VNPI score may be a useful adjunct in therapeutic decision making, but multiple attempts at independently validating the utility of this risk stratification scheme have not been consistent. Criticism of the USC/VNPI is that was applied to the same population in whom it was derived, the long treatment period (1972 to 1995), non-routine tissue processing, and extremely low rates of recurrence in the lowest risk group that have not been confirmed by other studies.

Rudloff et al. [49], from Memorial Sloan Kettering Cancer Center (MSKCC) from 1991 to 2006, identified 1868 consecutive women treated with BCS for DCIS. A multivariate Cox proportional hazards model was constructed using the 1681 women in whom data was complete. Ten clinical, pathologic, and treatment variables (age, family history, clinical presentation, margin status/width, size/volume of disease, nuclear grade, necrosis, time period, RT, and ET) were built into a nomogram estimating probability of IBTR at five and ten years after BCS. The model was validated for discrimination and calibration using bootstrap resampling. The DCIS nomogram for prediction of five- and ten-year IBTR probabilities demonstrated good calibration and discrimination, with a concordance index of $\mathbf{0 . 7 0 4}$ (bootstrap corrected, 0.688 ) and a concordance probability estimate of 0.686 . Factors with the greatest influence on risk of IBTR in the model included 
adjuvant RT or endocrine therapy, age, margin status, number of excisions, and treatment time period. This tool may assist in individual decision making regarding various treatment options and help avoid over- and under treatment of noninvasive breast cancer [49].

Yi et al. [50], retrospectively identified 794 patients with a diagnosis of DCIS who had undergone BCS from 1990 through 2007 at the MD Anderson Cancer Center (MDACC). Clinical and pathologic factors and the performance of the MSKCC nomogram for prediction of IBTR were assessed for 734 patients who had complete data. There was a marked difference with respect to tumor grade, prevalence of necrosis, initial presentation, final margins, and receipt of ET between the two cohorts. The biggest difference was that more patients received radiation in the MDACC cohort ( $75 \%$ at MDACC vs $49 \%$ at MSKCC; $\mathrm{p}<0.001$ ). Follow-up time in the MDACC cohort was longer than in the MSKCC cohort (median 7.1 years vs 5.6 years), and the recurrence rate was lower in the MDACC cohort $(7.9 \%$ vs $11 \%)$. The median five-year probability of recurrence was $5 \%$, and the median 10 -year probability of recurrence was $7 \%$. The nomogram for prediction of five- and ten-year IBTR probabilities demonstrated imperfect calibration and discrimination, with a concordance index of 0.63 . The authors concluded that predictive models for IBTR in patients with DCIS who were treated with BCS are imperfect [50].

Collins et al. [51], reviewed slides of patients with unilateral DCIS treated with BCT. Regression methods were used to estimate risks of local recurrence. The MSKCC DCIS nomogram was applied to the study population to compare the nomogram-predicted and observed local recurrence at five and ten years. The 495 patients in our study were grouped into quartiles and octiles to compare observed and nomogram-predicted local recurrence. The five-year absolute risk of recurrence for lowest and highest quartiles was $4.8 \%$ and 33.1\% (95 \% CI 3.1 6.4 and $24.2-40.9$, respectively; $\mathrm{p}<0.0001$ ). The overall correlation between ten-year nomogram-predicted recurrences and observed recurrences was 0.95 . Compared with observed ten-year local recurrence rates, the risk estimates provided by the nomogram showed good correlation, and reasonable discrimination with a c-statistic of $\mathbf{0 . 6 8}$. The authors concluded that the MSKCC DCIS nomogram provided good prediction of the five- and ten-year local recurrence when applied to a population of patients with DCIS treated with BCT in a community-based practice [51].

Sweldens et al. [52], from 1973 to 2010, identified 467 patients who were treated with BCS for DCIS at the University Hospital Leuven. Clinical, pathologic, and treatment parameters of all patients were used to create a multivariable model. The predictive value of the model was evaluated using the concordance index (C-index) and concordance probability estimate (CPE). Multiple imputation was used to account for missing data to allow the MSKCC model to be tested on 467 patients. The median follow-up was 7.2 years, with 48 women who developed an IBTR. Omission of adjuvant ET, younger age, and positive or close surgical margins were significantly associated with an increased risk of IBTR. 
The bootstrap-corrected C-index for ten-year prediction by our own model was 0.63 and the $\mathrm{CPE}$ was 0.61 . The $\mathrm{C}$-index and $\mathrm{CPE}$ for the ten-year relapse probabilities predicted by the MSKCC nomogram were 0.66 and 0.61 , respectively. Despite the small number of events, the need for multiple imputation, and few patients without RT, the MSKCC nomogram performance was somewhat better than our model. This shows that the MSKCC nomogram is externally valid [52].

In conclusion the MSKCC nomogram is externally validated, can risk stratify in various patient populations, and has excellent calibration. The discrimination across studies measured by the $\mathrm{C}$-index is around 0.6 to 0.7 which is comparable to the Oncotype Recurrence Score (0.69), the Gail model (0.58), and the adjuvant online (0.56). The nomogram uses readily available variables to provide individualized risk estimate. Both the USC/VNPI and MSKCC nomogram do not identify a low-risk subset of patients that require excision only so the applicability in every day practice is limited in counseling patients and it should not be used in guiding treatment recommendations.

Solin et al. in 2011 [53], presented the results of a trial evaluating the prognostic efficacy of Oncotype DCIS, a 12-gene molecular assay modeled on Oncotype DX. As with Oncotype DX, Oncotype DCIS stratifies patients into three score-based tiers: low risk (<39); intermediate risk (39 to 54$)$; and high-risk $(\geq 55)$. Using a new, pre-specified algorithm that had been optimized for DCIS gene expression, tissue samples from 650 women in the ECOG-ACRIN E5194 trial were tested, and higher Oncotype DCIS Score was found to be associated with increased likelihood of both overall (invasive and noninvasive, HR 2.34, CI $1.15-4.59, \mathrm{p}=$ 0.02 ) and invasive-only ( $\mathrm{HR} 3.73$, CI $1.34-9.82, \mathrm{p}=0.01$ ) ipsilateral breast events at ten years. Nonetheless, since the ECOG-ACRIN E5194 cohort was, by design, biased toward low-risk DCIS phenotypes, additional validation was sought [53].

Rakovitch et al. in 2015 [54], validated Oncotype DCIS in a cohort of 2,720 women with a more diverse distribution of DCIS clinical and pathologic features and who had been treated with BCS $+/-$ RT. The DCIS score was associated with increased risk of both invasive (HR 1.78, CI $1.03-3.05, \mathrm{p}=0.04$ ) and noninvasive (HR 2.43, CI $1.31-4.42, \mathrm{p}=0.005$ ) local recurrence regardless of ER status, though, $94.7 \%$ of the women had ER+ disease. Oncotype DCIS holds promise as a clinical decision-making tool in the management of DCIS, but prospective validation is needed [54].

The DCISionRT is a biological signature that calculates an individualized Decision Score (DS) that was developed and cross-validated in 526 DCIS patients treated with BCS \pm RT [55]. The relationship was assessed between DS and ten-year risk of invasive breast cancer (IBC) or any ipsilateral breast event (IBE), including IBC or DCIS. RT benefit was evaluated by risk group and as a function of DS. The DS was significantly associated with IBC and IBE risk, HR of 4.2 and 3.1, respectively. For patients treated without RT, the DS recognized a low risk group with a ten-year IBC risk of $4 \%$ (7\% IBE) and an elevated risk group with IBC risk of $15 \%$ (23\% IBE). In the analysis of the DS and RT by group, the ele- 
vated risk group received significant RT benefit, $\mathrm{HR}$ of 0.3 for IBC and IBE. In a clinical and pathologically low-risk subset, the DS reclassified $42 \%$ of patients into the elevated risk group. In an interaction analysis of DS and RT, patients with elevated DS had significant RT benefit over baseline. The authors concluded that the DS was prognostic for risk and predicted RT benefit for DCIS patients. It identified a clinically meaningful low-risk group and a group with elevated ten-year risks that received substantial RT benefit over baseline [55].

\section{Conclusion}

The surgical and adjuvant management of DCIS has advanced significantly in the last couple of decades, even so, surgeons, medical oncologist, and radiation oncologists, still depend on conventional clinical and pathologic risk factors to make management decisions. As our knowledge of the biology of DCIS continues to improve our goal in the management of DCIS is to prevent either under-treatment or over-treatment. In this paper, we will review the incidence and management options of DCIS. The need to change the views of the risk of DCIS and to avoid overtreatment is crucial, as over-treatment leads to physical and emotional harm for patients. Precisely, knowing when a lesion could be or will not be life-threatening necessitates a comprehensive understanding of the progression and evolution of DCIS in order to individualize care of these patients.

\section{Conflicts of Interest}

The authors declare no conflicts of interest regarding the publication of this paper.

\section{References}

[1] Bane, A. (2013) Ductal Carcinoma in Situ: What the Pathologist Needs to Know and Why. International Journal of Breast Cancer, 2013, Article ID: 914053. https://doi.org/10.1155/2013/914053

[2] Allegra, C.J., et al. (2010) National Institutes of Health State-of-the-Science Conference Statement: Diagnosis and Management of Ductal Carcinoma in Situ September 22-24, 2009. Journal of the National Cancer Institute, 102, 161-169. https://doi.org/10.1093/jnci/djp485

[3] Eusebi, V., et al. (1994) Long-Term Follow-Up of in Situ Carcinoma of the Breast. Seminars in Diagnostic Pathology, 11, 223-235.

[4] Collins, L.C., et al. (2005) Outcome of Patients with Ductal Carcinoma in Situ Untreated after Diagnostic Biopsy: Results from the Nurses' Health Study. Cancer, 103, 1778-1784. https://doi.org/10.1002/cncr.20979

[5] Siegel, R.L., Miller, K.D. and Jemal, A. (2020) Cancer Statistics, 2020. CA: A Cancer Journal for Clinicians, 70, 7-30. https://doi.org/10.3322/caac.21590

[6] Altekruse, S.F., Krapcho, M., et al. (2010) SEER Cancer Statistics Review, 1975-2007, N.C. Institute, Bethesda.

[7] Ernster, V.L., et al. (2002) Detection of Ductal Carcinoma in Situ in Women Undergoing Screening Mammography. Journal of the National Cancer Institute, 94, 1546-1554. https://doi.org/10.1093/jnci/94.20.1546 
[8] Bleyer, A. and Welch, H.G. (2012) Effect of Three Decades of Screening Mammography on Breast-Cancer Incidence. The New England Journal of Medicine, 367, 1998-2005. https://doi.org/10.1056/NEJMoa1206809

[9] Esserman, L.J., Thompson, I.M. and Reid, B. (2013) Overdiagnosis and Overtreatment in Cancer: An Opportunity for Improvement. JAMA, 310, 797-798. https://doi.org/10.1001/jama.2013.108415

[10] Frank, T.S., et al. (2002) Clinical Characteristics of Individuals with Germline Mutations in BRCA1 and BRCA2: Analysis of 10,000 Individuals. Journal of Clinical Oncology, 20, 1480-1490. https://doi.org/10.1200/JCO.2002.20.6.1480

[11] Anderson, B.O., Calhoun, K.E. and Rosen, E.L. (2006) Evolving Concepts in the Management of Lobular Neoplasia. Journal of the National Comprehensive Cancer Network, 4, 511-522. https://doi.org/10.6004/jnccn.2006.0041

[12] Stuart, K.E., et al. (2015) Long-Term Outcomes of Ductal Carcinoma in Situ of the Breast: A Systematic Review, Meta-Analysis and Meta-Regression Analysis. BMC Cancer, 15, Article No. 890. https://doi.org/10.1186/s12885-015-1904-7

[13] Simpson, T., Thirlby, R.C. and Dail, D.H. (1992) Surgical Treatment of Ductal Carcinoma in Situ of the Breast. 10- to 20-Year Follow-Up. Archives of Surgery, 127, 468-472. https://doi.org/10.1001/archsurg.1992.01420040114020

[14] Wanebo, H.J., Huvos, A.G. and Urban, J.A. (1974) Proceedings: Treatment of Minimal Breast Cancer. Cancer, 33, 349-357.

https://doi.org/10.1002/1097-0142(197402)33:2<349::AID-CNCR2820330208>3.0.C $\underline{\mathrm{O} ; 2-4}$

[15] Millis, R.R. and Thynne, G.S. (1975) In Situ Intraduct Carcinoma of the Breast: A Long Term Follow-Up Study. British Journal of Surgery, 62, 957-962. https://doi.org/10.1002/bjs.1800621209

[16] Tunon-de-Lara, C., et al. (2010) Ductal Carcinoma in Situ of the Breast in Younger Women: A Subgroup of Patients at High Risk. European Journal of Surgical Oncology, 36, 1165-1171. https://doi.org/10.1016/j.ejso.2010.09.001

[17] Owen, D., et al. (2013) Outcomes in Patients Treated with Mastectomy for Ductal Carcinoma in Situ. International Journal of Radiation Oncology, Biology, Physics, 85, e129-e134. https://doi.org/10.1016/j.ijrobp.2012.10.020

[18] Lara, J.F., et al. (2003) The Relevance of Occult Axillary Micrometastasis in Ductal Carcinoma in Situ: A Clinicopathologic Study with Long-Term Follow-Up. Cancer, 98, 2105-2113. https://doi.org/10.1002/cncr.11761

[19] Sunshine, J.A., et al. (1985) Breast Carcinoma in Situ. A Retrospective Review of 112 Cases with a Minimum 10 Year Follow-Up. The American Journal of Surgery, 150, 44-51. https://doi.org/10.1016/0002-9610(85)90008-X

[20] Akashi-Tanaka, S., Nanasawa, T., Matsuo, K., Hasegawa, T., Tsuda, H., et al. (2000) Treatment of Noninvasive Carcinoma: Fifteen-Year Results at the National Cancer Center Hospital in Tokyo. Breast Cancer, 7, 341-344. https://doi.org/10.1007/BF02966402

[21] Tuttle, T.M., et al. (2009) Increasing Rates of Contralateral Prophylactic Mastectomy among Patients with Ductal Carcinoma in Situ. Journal of Clinical Oncology, 27, 1362-1367. https://doi.org/10.1200/JCO.2008.20.1681

[22] Yao, K., et al. (2010) Trends in Contralateral Prophylactic Mastectomy for Unilateral Cancer: A Report from the National Cancer Data Base, 1998-2007. Annals of Surgical Oncology, 17, 2554-2562. https://doi.org/10.1245/s10434-010-1091-3 
[23] Wapnir, I.L., et al. (2011) Long-Term Outcomes of Invasive Ipsilateral Breast Tumor Recurrences after Lumpectomy in NSABP B-17 and B-24 Randomized Clinical Trials for DCIS. Journal of the National Cancer Institute, 103, 478-488. https://doi.org/10.1093/jnci/djr027

[24] Silverstein, M.J., et al. (1992) Duct Carcinoma in Situ: 227 Cases without Microinvasion. European Journal of Cancer, 28, 630-634. https://doi.org/10.1016/S0959-8049(05)80114-5

[25] Cuzick, J., et al. (2011) Effect of Tamoxifen and Radiotherapy in Women with Locally Excised Ductal Carcinoma in Situ: Long-Term Results from the UK/ANZ DCIS Trial. The Lancet Oncology, 12, 21-29. https://doi.org/10.1016/S1470-2045(10)70266-7

[26] Donker, M., et al. (2013) Breast-Conserving Treatment with or without Radiotherapy in Ductal Carcinoma in Situ: 15-Year Recurrence Rates and Outcome after a Recurrence, from the EORTC 10853 Randomized Phase III Trial. Journal of Clinical Oncology, 31, 4054-4059. https://doi.org/10.1200/JCO.2013.49.5077

[27] Warnberg, F., et al. (2014) Effect of Radiotherapy after Breast-Conserving Surgery for Ductal Carcinoma in Situ: 20 Years Follow-Up in the Randomized SweDCIS Trial. Journal of Clinical Oncology, 32, 3613-3618. https://doi.org/10.1200/JCO.2014.56.2595

[28] Fisher, B., et al. (1998) Lumpectomy and Radiation Therapy for the Treatment of Intraductal Breast Cancer: Findings from National Surgical Adjuvant Breast and Bowel Project B-17. Journal of Clinical Oncology, 16, 441-452. https://doi.org/10.1200/JCO.1998.16.2.441

[29] Early Breast Cancer Trialists' Collaborative G., et al. (2010) Overview of the Randomized Trials of Radiotherapy in Ductal Carcinoma in Situ of the Breast. Journal of the National Cancer Institute Monograph, 2010, 162-177. https://doi.org/10.1093/jncimonographs/lgq039

[30] Darby, S.C., et al. (2005) Long-Term Mortality from Heart Disease and Lung Cancer after Radiotherapy for Early Breast Cancer: Prospective Cohort Study of about 300,000 Women in US SEER Cancer Registries. The Lancet Oncology, 6, 557-565. https://doi.org/10.1016/S1470-2045(05)70251-5

[31] Allred, D.C., et al. (2012) Adjuvant Tamoxifen Reduces Subsequent Breast Cancer in Women with Estrogen Receptor-Positive Ductal Carcinoma in Situ: A Study Based on NSABP Protocol B-24. Journal of Clinical Oncology, 30, 1268-1273. https://doi.org/10.1200/JCO.2010.34.0141

[32] Solin, L.J., et al. (2015) Surgical Excision without Radiation for Ductal Carcinoma in Situ of the Breast: 12-Year Results From the ECOG-ACRIN E5194 Study. Journal of Clinical Oncology, 33, 3938-3944. https://doi.org/10.1200/JCO.2015.60.8588

[33] McCormick, B., et al. (2015) RTOG 9804: A Prospective Randomized Trial for Good-Risk Ductal Carcinoma in Situ Comparing Radiotherapy with Observation. Journal of Clinical Oncology, 33, 709-715. https://doi.org/10.1200/JCO.2014.57.9029

[34] Margolese, R.G., et al. (2016) Anastrozole versus Tamoxifen in Postmenopausal Women with Ductal Carcinoma in Situ Undergoing Lumpectomy plus Radiotherapy (NSABP B-35): A Randomised, Double-Blind, Phase 3 Clinical Trial. The Lancet, 387, 849-856. https://doi.org/10.1016/S0140-6736(15)01168-X

[35] Forbes, J.F., et al. (2016) Anastrozole versus Tamoxifen for the Prevention of Locoregional and Contralateral Breast Cancer in Postmenopausal Women with Locally Excised Ductal Carcinoma in Situ (IBIS-II DCIS): A Double-Blind, Randomised 
Controlled Trial. The Lancet, 387, 866-873.

https://doi.org/10.1016/S0140-6736(15)01129-0

[36] Cox, C.E., et al. (1998) Guidelines for Sentinel Node Biopsy and Lymphatic Mapping of Patients with Breast Cancer. Annals of Surgery, 227, 645-651.

[37] Klauber-DeMore, N., et al. (2000) Sentinel Lymph Node Biopsy: Is It Indicated in Patients with High-Risk Ductal Carcinoma-in-Situ and Ductal Carcinoma-in-Situ with Microinvasion? Annals of Surgical Oncology, 7, 636-642. https://doi.org/10.1007/s10434-000-0636-2

[38] Francis, A.M., et al. (2015) Is Sentinel Lymph Node Dissection Warranted for Patients with a Diagnosis of Ductal Carcinoma In Situ? Annals of Surgical Oncology, 22, 4270-4279. https://doi.org/10.1245/s10434-015-4547-7

[39] Lyman, G.H., Temin, S., Edge, S.B., Newman, L.A., Turner, R.R., Weaver, D.L., Benson III, A.B., Bosserman, L.D., Burstein, H.J., Cody III, H., Hayman, J., Perkins, C.L., Podoloff, D.A. and Giuliano, A.E. (2014) Sentinel Lymph Node Biopsy for Patients with Early-Stage Breast Cancer: American Society of Clinical Oncology Clinical Practice Guideline Update. Journal of Clinical Oncology, 32, 1365-1383. https://doi.org/10.1200/JCO.2013.54.1177

[40] Hwang, E.S., et al. (2019) The COMET (Comparison of Operative versus Monitoring and Endocrine Therapy) Trial: A Phase III Randomised Controlled Clinical Trial for Low-Risk Ductal Carcinoma in Situ (DCIS). BMJ Open, 9, e026797. https://doi.org/10.1136/bmjopen-2018-026797

[41] Francis, A. A Phase III Trial of Surgery versus Active Monitoring for Low Risk Ductal Carcinoma in Situ (DCIS).

https://www.birmingham.ac.uk/research/activity/mds/trials/crctu/trials/loris/index. $\underline{\text { aspx }}$

[42] Wesseling, J. https://clinicaltrials.gov/ct/show/NCT02492607 http://www.eortc.be/clinicaltrials/Details.asp?Protocol=1401\&B1=Search https://www.boogstudycenter.nl/studie/276/lord.html

[43] Pilewskie, M., et al. (2016) Women with Low-Risk DCIS Eligible for the LORIS Trial after Complete Surgical Excision: How Low Is Their Risk after Standard Therapy? Annals of Surgical Oncology, 23, 4253-4261. https://doi.org/10.1245/s10434-016-5595-3

[44] Kanbayashi, C., Thompson, A.M., Hwang, E.-S.S., Partridge, A.H., Rea, D.W., Wesseling, J., Shien, T., Mizutani, T., Shibata, T. and Iwata, H. (2019) The International Collaboration of Active Surveillance Trials for Low-Risk DCIS (LORIS, LORD, COMET, LORETTA). Journal of Clinical Oncology, 37, TPS603. https://doi.org/10.1200/JCO.2019.37.15_suppl.TPS603

[45] Boyages, J., Delaney, G. and Taylor, R. (1999) Predictors of Local Recurrence after Treatment of Ductal Carcinoma in Situ: A Meta-Analysis. Cancer, 85, 616-628. https://doi.org/10.1002/(SICI)1097-0142(19990201)85:3<616::AID-CNCR12>3.0.C $\underline{\mathrm{O} ; 2-7}$

[46] Lagios, M.D., et al. (1989) Mammographically Detected Duct Carcinoma in Situ. Frequency of Local Recurrence Following Tylectomy and Prognostic Effect of Nuclear Grade on Local Recurrence. Cancer, 63, 618-624. https://doi.org/10.1002/1097-0142(19890215)63:4<618::AID-CNCR2820630403>3.0 .CO;2-J

[47] Silverstein, M.J., et al. (1995) Prognostic Classification of Breast Ductal Carcinoma-in-Situ. The Lancet, 345, 1154-1157.

https://doi.org/10.1016/S0140-6736(95)90982-6 
[48] Silverstein, M.J. and Lagios, M.D. (2010) Choosing Treatment for Patients with Ductal Carcinoma in Situ: Fine Tuning the University of Southern California/Van Nuys Prognostic Index. Journal of the National Cancer Institute Monograph, 2010, 193-196. https://doi.org/10.1093/jncimonographs/lgq040

[49] Rudloff, U., et al. (2010) Nomogram for Predicting the Risk of Local Recurrence after Breast-Conserving Surgery for Ductal Carcinoma in Situ. Journal of Clinical Oncology, 28, 3762-3769. https://doi.org/10.1200/JCO.2009.26.8847

[50] Yi, M., et al. (2012) Evaluation of a Breast Cancer Nomogram for Predicting Risk of Ipsilateral Breast Tumor Recurrences in Patients with Ductal Carcinoma in Situ after Local Excision. Journal of Clinical Oncology, 30, 600-607. https://doi.org/10.1200/JCO.2011.36.4976

[51] Collins, L.C., et al. (2015) Risk Prediction for Local Breast Cancer Recurrence among Women with DCIS Treated in a Community Practice: A Nested, Case-Control Study. Annals of Surgical Oncology, 22, S502-S508. https://doi.org/10.1245/s10434-015-4641-x

[52] Sweldens, C., et al. (2014) Local Relapse after Breast-Conserving Therapy for Ductal Carcinoma in Situ: A European Single-Center Experience and External Validation of the Memorial Sloan-Kettering Cancer Center DCIS Nomogram. The Cancer Journal, 20, 1-7. https://doi.org/10.1097/PPO.0000000000000025

[53] Solin, L.J., et al. (2013) A Multigene Expression Assay to Predict Local Recurrence Risk for Ductal Carcinoma in Situ of the Breast. Journal of the National Cancer Institute, 105, 701-710. https://doi.org/10.1093/jnci/djt067

[54] Rakovitch, E., et al. (2015) A Population-Based Validation Study of the DCIS Score Predicting Recurrence Risk in Individuals Treated by Breast-Conserving Surgery Alone. Breast Cancer Research and Treatment, 152, 389-398. https://doi.org/10.1007/s10549-015-3464-6

[55] Bremer, T., et al. (2018) A Biological Signature for Breast Ductal Carcinoma in Situ to Predict Radiotherapy Benefit and Assess Recurrence Risk. Clinical Cancer Research, 24, 5895-5901. https://doi.org/10.1158/1078-0432.CCR-18-0842 